\title{
CONSTRAINING THE LORENTZ FACTOR OF A RELATIVISTIC SOURCE FROM ITS THERMAL EMISSION
}

\author{
Yuan-Chuan Zou ${ }^{1,2}$, K. S. Cheng ${ }^{2}$, and F. Y. Wang ${ }^{2,3}$ \\ ${ }^{1}$ School of Physics, Huazhong University of Science and Technology, Wuhan 430074, China; zouyc@ @ust.edu.cn \\ ${ }^{2}$ Department of Physics, University of Hong Kong, Hong Kong, China \\ ${ }^{3}$ School of Astronomy and Space Science, Nanjing University, Nanjing 210093, China \\ Received 2014 December 9; accepted 2015 January 24; published 2015 February 17
}

\begin{abstract}
We propose a direct and simple method to measure the Lorentz factor of relativistically expanding objects such as gamma-ray bursts (GRBs). Only three measurements, i.e., the thermal component of the emission, the distance, and the variable timescale of the light curve, are used. When the uncertainties are considered, we will obtain a lower limit of the Lorentz factor instead. We apply this method to GRB 090618 and get a lower limit of 22 for the Lorentz factor. This method can be used for any relativistically moving objects, such as GRBs and soft gamma-ray repeaters.
\end{abstract}

Key words: gamma-ray burst: general - radiation mechanisms: thermal - relativistic processes

\section{INTRODUCTION}

For a relativistically moving object, it is crucial to know the Lorentz factor $(\Gamma)$. In the context of astrophysical objects, there are several phenomena that are believed to be associated with relativistic motions, such as gamma-ray bursts (GRBs; Piran 2004), active galactic nuclei (AGNs; Ghisellini et al. 1993), and soft gamma-ray repeaters (SGRs; Norris et al. 1991). Especially for GRBs, the Lorentz factors of jets are believed to be about a few hundred.

The identification of high $\Gamma$ is from the compactness problem (Rees 1967). There are several methods used to constrain the Lorentz factor of the relativistic motion, such as superluminal motion for AGNs (Vermeulen \& Cohen 1994), the afterglow deceleration time of GRBs (Meszaros \& Rees 1997), the thermal emission from a standard fireball at the photosphere radius (Nakar et al. 2005; Pe'er et al. 2007), the compactness problem for the observed high energy photons (Fenimore et al. 1993; Lithwick \& Sari 2001), and the quiet period of the light curves (Zou \& Piran 2010). However, these methods are either model dependent (or parameter dependent; Vermeulen \& Cohen 1994; Meszaros \& Rees 1997; Nakar et al. 2005; Pe'er et al. 2007) or only upper/lower limits can be obtained (Fenimore et al. 1993; Zou \& Piran 2010).

Our method can directly derive (or constrain the lower limit of) $\Gamma$ if the thermal emission is identified, while this thermal component has been observed in many cases, such as GRB 090618 (Page et al. 2011), GRB 090902B (Pe'er et al. 2012), and GRB 100724B (Guiriec et al. 2011). Fan et al. (2012) suggested that the thermal component may even dominate the prompt emission in some GRBs (see also Vereshchagin 2014). We mainly concentrate on the GRBs, because the GRB jets are mostly believed to be pointing toward the observers. Considering the beaming effect of the relativistic jet, spherical geometry can be safely taken only if the jet opening angle $\theta_{j}>\frac{1}{\Gamma}$. The general scenario of the GRB thermal emission is described by Toma et al. (2011): multiple conical shells are expanding, which produce the thermal pulses, while the internal shocks from the collision of these shells produce the nonthermal emission at larger radii. The superposition of these two kinds of emissions is the observed spectrum. Here we concentrate on the thermal component to derive the Lorentz factor of these shells. We describe the basic concept in
Section 2, apply the method to GRBs in section Section 3, and discuss the results in Section 4.

\section{BASIC CONCEPTS}

For a relativistically expanding shell, the thermal photons are coupled with the electrons at the beginning. With the expansion of the relativistic flow (either spherical or conical in shape), it becomes optically thin at the photosphere, where the optical depth drops down to unity. At this radius, almost all the thermal photons escape with their luminosity following the blackbody spectrum. Beyond this radius, as no more photons are produced, emission drops down quickly and can be neglected, the energy stored in the plasma will be released by the internal or external shocks at larger radii, where nonthermal emission (synchrotron radiation for instance) dominates. Therefore, the observed thermal emission can be taken as blackbody emission at the photosphere. ${ }^{4}$

Because of the beaming effect, for a blackbody sphere expanding with Lorentz factor $\Gamma(\Gamma \gg 1)$, the received flux is mainly emitted from the region within $\theta \leqslant \frac{1}{\Gamma}$, where $\theta$ is the angle starting from the line of sight, as shown in Figure 1. The blackbody flux we receive satisfies

$$
F_{\text {thermal }}^{\text {obs }} \leqslant F_{\mathrm{bb}} \simeq \frac{\sigma\left[(1+z) T_{\mathrm{obs}}\right]^{4} 4 \pi\left(\frac{R_{\mathrm{ph}}}{\Gamma}\right)^{2}}{4 \pi D_{L}^{2}},
$$

where $F_{\text {thermal }}^{\text {obs }}$ is the observed flux of the thermal component, $F_{\mathrm{bb}}$ is the observed flux by assuming the radiation is from a blackbody in thermal equilibrium, $\sigma=5.67 \times 10^{-5} \mathrm{erg} \mathrm{cm}^{-2} \mathrm{~s}^{-1} \mathrm{~K}^{-4}, z$ is the redshift, $T_{\mathrm{obs}}$ is the observed temperature, $R_{\mathrm{ph}}$ is the photosphere radius of the

\footnotetext{
4 There is another possibility, namely, that in the optically thick stage photons coupled with electrons are no longer produced. With the expansion of the relativistic shells, the shape of the spectrum becomes a Compton spectrum (Vurm et al. 2013). The number density of the photons is then smaller than that of a blackbody emission. Consequently, the luminosity at the photosphere is lower than that of a blackbody with the same temperature. This leads to a lower limit for the Lorentz factor. Though, in principle, this kind of thermal emission can be distinguished from the blackbody emission (Plank spectrum) by the spectral shape. It is not easy to distinguish in real data. The combination leads to a lower limit rather than a certain value, which is shown in Equation (1). Whether or not the Compton spectrum is being distinguished from the Plank spectrum, Equation (1) splits into either an equality or a lower limit.
} 


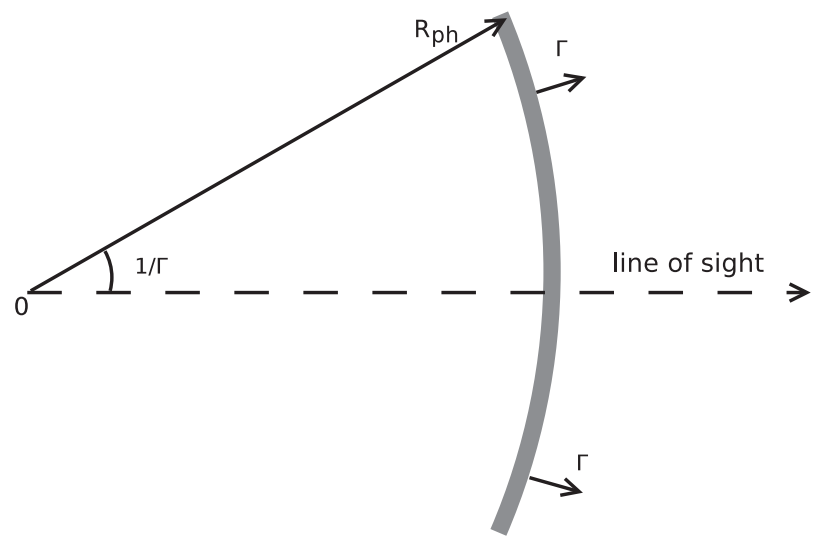

Black-body radiating shell

Figure 1. Sketch for the conical radiating shell at the photosphere, which is moving with Lorentz factor $\Gamma$. Because of the beaming effect, roughly only the region inside $1 / \Gamma$ can be seen by the observer. This determines the timescale of the thermal pulse.

expanding fireball, and $D_{L}$ is the luminosity distance. $F_{\text {thermal }}^{\text {obs }} \leqslant F_{\text {bb }}$ comes from the fact that the Thompson scattering photosphere can be larger than the absorption/radiation photosphere (see Vurm et al. 2013, for more details). Notice that the equation is also applicable for a conical jet with an opening angle $\theta_{j}>\frac{1}{\Gamma}$ when the line of sight is inside the jet angle.

Because almost all of the thermal photons in the plasma will suddenly be radiated at the photosphere, the observed duration of this emission is generally dominated by the angular timescale (Piran 1999), $\delta t_{\mathrm{ang}} \simeq \frac{(1+z) R_{\mathrm{ph}}}{2 \Gamma^{2} c}$, where $c$ is the speed of light. In some cases, the angular timescale is just a lower limit, e.g., for the thick shell case before the spreading radius, the observed timescale is dominated by the thickness of the shell, or several thermal pulses are too close to be distinguished, both cases imply $\delta t_{\oplus} \geqslant \delta t_{\text {ang }}$, i.e.,

$$
\delta t_{\oplus} \geqslant \frac{(1+z) R_{\mathrm{ph}}}{2 \Gamma^{2} c} .
$$

Combining Equations (1) and (2), we get the lower limit for the bulk Lorentz factor:

$$
\Gamma \geqslant\left[\frac{F_{\text {thermal }}^{\text {obs }} D_{L}^{2}}{(1+z)^{2} \sigma T_{\text {obs }}^{4}\left(2 c \delta t_{\oplus}\right)^{2}}\right]^{\frac{1}{2}} .
$$

Because all of the variables on the right-hand side are observables, the $\Gamma$ is then a directly measurable quantity, by measuring the spectrum of the thermal component and the redshift of the object. The equality in the equation above applies in the ideal condition: the emitting region is geometrically thin, and is in thermal equilibrium.

There is another constraint from the fact that the thermal component is mainly released at the radius where the shell becomes optically thin, i.e., the optical depth $\tau=1$. This determines the radius of the photosphere (Daigne \& Mochkovitch 2002; Mészáros et al. 2002)

$$
R_{\mathrm{ph}}=\frac{L \sigma_{T}}{8 \pi \Gamma^{3} m_{p} c^{3}}
$$

where $L$ is the kinetic luminosity of the ejecta, which is taken as cold when the thermal photons are emitted, $\sigma_{T}$ is the Thompson cross-section and $m_{p}$ is the mass of the proton. Pe'er et al. (2007) considered this relation together with the blackbody radiation luminosity (i.e., Equation (1)) to derive the initial Lorentz factor without considering the timescale (i.e., Equation (2)). ${ }^{5}$ One should consider the consistency between the three equations: Equations (1), (2), and (4), but keeping in mind the uncertainties of Equation (4) arising from the facts: (1) the efficiency of conversion of the kinetic energy to the observed $\gamma$-ray energy is not known, it may include the Poynting flux making the efficiency even more uncertain; (2) the amount of electron-positron pairs is not known, which will affect the optical depth; (3) the matter should be fully ionized, otherwise another parameter characterizing the ionization should be introduced; (4) Equation (4) is only suitable when the Thompson scattering is the dominant cause of opacity; (5) $F_{\text {thermal }}^{\text {obs }}=F_{\mathrm{bb}}$ is assumed. In the optimistic condition, one can combine Equations (4) and (1) to get the Lorentz factor by introducing an efficiency parameter $Y \equiv L / L_{\gamma}\left(L_{\gamma}\right.$ is the observed $\gamma$-ray luminosity; Pe'er et al. 2007)

$$
\Gamma=\left[\frac{\sigma \sigma_{T}(1+z)^{2} Y L_{\gamma} T_{\mathrm{obs}}^{2}}{8 \pi m_{p} c^{3} D_{L} F_{\text {thermal }}^{\text {obs } 1 / 2}}\right]^{1 / 4} .
$$

\section{APPLICATION TO GRBS}

Taking the typical values of GRBs into Equation (3), one gets the scaling law

$$
\Gamma \geqslant 110 F_{\text {thermal, }-6}^{\text {obs } \frac{1}{2}} D_{\mathrm{L}, 28}\left(\frac{1+z}{2}\right)^{-1} T_{\mathrm{obs}, 8}^{-2} \delta t_{\oplus,-2}^{-1} .
$$

The conventional notation $Q=Q_{x} \times 10^{x}$ is used throughout this letter. The greatest uncertainty here is the choice of timescale $\delta t_{\oplus} \sim 0.01 \mathrm{~s}$, as the variability of the thermal component has not been identified in such small time bins (Page et al. 2011; Guiriec et al. 2013; Burgess et al. 2014). Though the observed typical timescale for the GRB pulses is of the order of $0.1 \mathrm{~s} \mathrm{(Nakar} \mathrm{\&} \mathrm{Piran} \mathrm{2002),} \mathrm{they} \mathrm{are} \mathrm{believed} \mathrm{to} \mathrm{be}$ from the internal shocks that occur farther out than the photospheric radius. Consequently, the timescale of the photosphere radius should be smaller. It may also be supported by the finding that the GRB pulses also contain millisecond variabilities (Walker et al. 2000).

There are several GRBs that were reported to have the thermal component in the prompt emission: GRB 060218 (Campana et al. 2006), GRB 090618 (Page et al. 2011), GRB 090902B (Pe'er et al. 2012), GRB 100316D (Starling et al. 2011), GRB 100724B (Guiriec et al. 2011), GRB 120323A (Guiriec et al. 2013), and more in a recent analysis (Burgess et al. 2014). However, most of them have no light curve of the thermal component, which prevents us from estimating the timescale. Among them, GRB 120323A is a strong short burst. It makes the time-resolved spectral analysis possible, which shows a thermal timescale less than $0.1 \mathrm{~s}$

\footnotetext{
5 Though they did not explicitly consider the angular timescale, when they argue that the thermal emission after the peak at around $8 \mathrm{~s}$ of GRB 970828 comes from the off-axis emission, the angular timescale shows that the off-axis emission could not last longer than $10 \mathrm{~s}$. In addition, if the followed thermal component was coming from the high latitude, such as Pe'er et al. (2007) claimed, the temperature decay with time should be $t^{-1}$ but not $t^{-0.51}$ as observed. Therefore, we conclude that the decaying timescale of seconds is not from the off-axis emission.
} 
(Guiriec et al. 2013). Unfortunately, the redshift of this burst is not known.

We take GRB 090618 as an example. It has been reported to have a thermal component, with $F_{\text {thermal }}^{\text {obs }} \simeq 10^{-6} \mathrm{erg} \mathrm{cm}^{-2} \mathrm{~s}^{-1}$, $z=0.54, \quad D_{L}=9.6 \times 10^{27} \mathrm{~cm}, \quad k T_{\text {obs }} \simeq 20 \mathrm{keV} \quad($ Page et al. 2011). The most uncertainty is in the timescale of the thermal pulses, while $\Gamma$ is sensitive to it. We choose $\delta t \sim 0.01 \mathrm{~s}$ as a typical timescale for the thermal pulses. Then the Lorentz factor is greater than $\sim 22$ by taking the values into Equation (6). The uncertainty of the timescale of the thermal component makes the constraint trivial. This constraint will be enhanced if the short time-resolved spectral analysis can be performed, just like GRB 120323A.

We may consider the consistency of this method with other methods in the Lorentz factor constraint. Taking the observed quantities into Equation (5), we get the Lorentz factor to be $130 Y^{1 / 4}$. This is consistent with the lower limit above (keep in mind, even if Equation (5) is not consistent with other methods, that might be because Equation (5) is not suitable for the reasons listed above). One can also estimate the Lorentz factor by the afterglow light curve (Meszaros \& Rees 1997). From the X-ray afterglow of GRB 090618, no peak was observed, which indicates that the peak time (deceleration time) should be smaller than $300 \mathrm{~s}$, as shown in the light curve (Dado \& Dar 2010). This upper limit of peak time (300 s) indicates a lower limit for the initial Lorentz factor of roughly 150, which was obtained by using Equation (1) of Lü et al. (2012), taking observed quantities and assuming an ambient number density $1 \mathrm{~cm}^{-3}$. Hence the direct Lorentz factor is also consistent with the estimation from the afterglow deceleration timescale. It should be consistent with the lower limit given by Lithwick \& Sari (2001). However, the required high energy photons from GRB 090426 were not reported. On the other hand, a lower limit is always consistent with another lower limit obtained by a different method.

Besides the prompt emission, one can apply our method to estimate the Lorentz factor from the thermal emission of the afterglow. Consequently, the Lorentz factor characterizes the afterglow rather than the prompt emission region. GRB 090618 has also been reported with a thermal component from the afterglow at hundreds of seconds (Page et al. 2011). However, it was in the steep decline stage (Zhang et al. 2006), which is mainly taken as indicating high latitude emission from the prompt radiating shell. Hence, this thermal component cannot be directly used to derive the Lorentz factor.

GRBs are widely known to be highly relativistic with $\Gamma \sim 100$ (Piran 2004). For a given $\Gamma \sim 100$, we can see from Equation (6) that the temperature $T_{\mathrm{obs}}$ must be relatively low (say roughly $<10 \mathrm{keV}$ ), otherwise, the thermal radiation will be extremely high and cannot be missed by the spacecraft. The relatively low temperature might be the reason why so few GRBs have been firmly determined to have the thermal component.

Notice that very few GRBs prompt emission are identified with a thermal component, and the thermal components are all identified with observed temperatures less than $10 \mathrm{keV}$. The main reason is that the current on-boarding satellite missions mainly cover relatively high energy bands (more than tens of $\mathrm{keV}$ ). It may be resolved by the lower band soft $\gamma$-ray telescopes scheduled to be launched, such as SVOM (Götz \& SVOM Collaboration 2012), JANUS (Falcone et al. 2009), and LOFT (Amati et al. 2015). After identifying the thermal component, one needs to identify the timescales of the thermal pulses, which means the time bin should be small enough to perform a spectral analysis. This is still challenging but might be available for strong soft bursts in the near future. Otherwise, only lower limits can be achieved.

\section{DISCUSSION}

To clarify, we emphasize the scenario for the thermal and nonthermal components in the following. The relativistic warm shells produced from the central engine are ejected stochastically. With the shells expanding spherically, the optical depth for the trapped thermal photons decreases. At the transparent radius, the shell emits almost all of the thermal photons, which are observed as thermal components. Each shell will produce one thermal pulse with a short timescale. The combination of any two shells produces one internal shock, which generally occurs at a larger radius, and produces the nonthermal component. This nonthermal component has a longer timescale. The superposition of these two components is what we observe. For the purpose of obtaining the Lorentz factor, we need to extract the thermal component. For GRBs, the thermal component should never be dominant. Otherwise, there will be more energy left in the plasma. This is related to the energy budget problem and the efficiency problem (Piran 2004). This makes the extraction of the thermal light curve more difficult.

In general, this method can be applied to any object radiating thermal emission, and with a relativistic velocity. There are several other objects to which this method may potentially be applicable, though shortcomings are obvious at the present stage. SGRs are also thought to be relativistic events (Cheng \& Wang 2003, for example). However, the blackbody emission in this case is mainly taken to be generated from the progenitor (Hurley et al. 2005). This method cannot be directly applied to AGNs because AGN jets are mainly pointing to other directions, they are all continuous jets, and there is no confirmed thermal radiation (Fossati et al. 1998). X-ray binaries are believed to have relativistic jets as well. However, the number of on-axis cases is very limited, and the thermal component is not easily distinguished as arising from the jet or from the disk. Tidal disruption events also have mildly relativistic ejecta. However, no associated thermal component has been identified. The low Lorentz factor may be beyond the restriction $1 / \Gamma \leqslant \theta_{j}$ and is not easy to obtain by our method. This method may also be applied to the GRB afterglow stage if there is a thermal component (Peng et al. 2014).

We also notice that it is applicable only if the following conditions are satisfied. (1) The jet opening angle should be greater than $1 / \Gamma$, otherwise the shortest timescale is not $R_{\mathrm{ph}} / 2 \Gamma^{2} c$. Fortunately, for the GRBs, the jet opening angle is generally greater than $1 / \Gamma$. (2) The line of sight should be inside the jet opening angle, and this is always the case for GRBs. (3) Though, in the GRB fireball scenario it is unlikely that the ejecta is still accelerating at the photosphere radius, in general, it is possible. Then the derived Lorentz factor is again a lower limit.

One can also constrain the Lorentz factor by other methods, especially for constraining the upper limits. By combining several methods, once the Lorentz factor is constrained in a very narrow range without inconsistency, that means the equality of Equation (3) can be applied. This indicates that, for such a burst, the thermal emission comes from a thin shell and no heating of the electrons occurred. 
We thank Prof. Kevin MacKeown for a critical reading of the manuscript. Y.C.Z. acknowledges helpful discussions with Tsvi Piran, Zigao Dai, Bing Zhang, Xuefeng Wu, Xiwei Liu, Weihua Lei, Rongfeng Shen, and Rodolfo Barniol Duran. This work is supported by the Chinese-Israeli Joint Research Project (grant No. 11361140349), the National Basic Research Program of China (973 Program, grant No. 2014CB845800), and the National Natural Science Foundation of China (grant No. U1231101). K.S.C. is supported by the CRT Grants of the Government of the Hong Kong SAR under HKUST4/ $\mathrm{CRF} / 13 \mathrm{G}$.

\section{REFERENCES}

Amati, L., Stratta, G., Atteia, J.-L., et al. 2015, arXiv:1501.02772

Burgess, J. M., Preece, R. D., Ryde, F., et al. 2014, ApJL, 784, L43

Campana, S., Mangano, V., Blustin, A. J., et al. 2006, Natur, 442, 1008

Cheng, K. S., \& Wang, X. Y. 2003, ApJL, 593, L85

Dado, S., \& Dar, A. 2010, ApJL, 708, L112

Daigne, F., \& Mochkovitch, R. 2002, MNRAS, 336, 127

Falcone, A. D., Burrows, D. N., Barthelmy, S., et al. 2009, Proc. SPIE, 7435, 743508

Fan, Y.-Z., Wei, D.-M., Zhang, F.-W., \& Zhang, B.-B. 2012, ApJL, 755, L6 Fenimore, E. E., Epstein, R. I., \& Ho, C. 1993, A\&AS, 97, 59

Fossati, G., Maraschi, L., Celotti, A., Comastri, A., \& Ghisellini, G. 1998, MNRAS, 299, 433
Ghisellini, G., Padovani, P., Celotti, A., \& Maraschi, L. 1993, ApJ, 407, 65 Götz, D. SVOM Collaboration 2012, MSAIS, 21, 162

Guiriec, S., Connaughton, V., Briggs, M. S., et al. 2011, ApJL, 727, L33

Guiriec, S., Daigne, F., Hascoët, R., et al. 2013, ApJ, 770, 32

Hurley, K., Boggs, S. E., Smith, D. M., et al. 2005, Natur, 434, 1098

Lithwick, Y., \& Sari, R. 2001, ApJ, 555, 540

Lü, J., Zou, Y.-C., Lei, W.-H., et al. 2012, ApJ, 751, 49

Mészáros, P., Ramirez-Ruiz, E., Rees, M. J., \& Zhang, B. 2002, ApJ, 578, 812

Meszaros, P., \& Rees, M. J. 1997, ApJ, 476, 232

Nakar, E., \& Piran, T. 2002, MNRAS, 330, 920

Nakar, E., Piran, T., \& Sari, R. 2005, ApJ, 635, 516

Norris, J. P., Hertz, P., Wood, K. S., \& Kouveliotou, C. 1991, ApJ, 366, 240

Page, K. L., Starling, R. L. C., Fitzpatrick, G., et al. 2011, MNRAS, 416, 2078

Pe'er, A., Ryde, F., Wijers, R. A. M. J., Mészáros, P., \& Rees, M. J. 2007, ApJL, 664, L1

Pe'er, A., Zhang, B.-B., Ryde, F., et al. 2012, MNRAS, 420, 468

Peng, F.-K., Liang, E.-W., Wang, X.-Y., et al. 2014, ApJ, 795, 155

Piran, T. 1999, PhR, 314, 575

Piran, T. 2004, RvMP, 76, 1143

Rees, M. J. 1967, MNRAS, 135, 345

Starling, R. L. C., Wiersema, K., Levan, A. J., et al. 2011, MNRAS, 411, 2792

Toma, K., Wu, X.-F., \& Mészáros, P. 2011, MNRAS, 415, 1663

Vereshchagin, G. 2014, IJMPD, 23, 1430003

Vermeulen, R. C., \& Cohen, M. H. 1994, ApJ, 430, 467

Vurm, I., Lyubarsky, Y., \& Piran, T. 2013, ApJ, 764, 143

Walker, K. C., Schaefer, B. E., \& Fenimore, E. E. 2000, ApJ, 537, 264

Zhang, B., Fan, Y. Z., Dyks, J., et al. 2006, ApJ, 642, 354

Zou, Y.-C., \& Piran, T. 2010, MNRAS, 402, 1854 